\title{
Increased FoxM1 expression is a target for metformin in the suppression of EMT in prostate cancer
}

\author{
YIRU WANG $^{1 *}$, BINWEI YAO $^{2 *}$, YU WANG $^{3,4}$, MINGBO ZHANG $^{1}$, SHUAI FU $^{1}$, \\ HANJING GAO ${ }^{1}$, RUIYUN PENG ${ }^{2}$, LINGQIANG ZHANG $^{5}$ and JIE TANG ${ }^{1}$
}

${ }^{1}$ Department of Ultrasound, Chinese PLA General Hospital, Beijing 100853; ${ }^{2}$ Department of Experimental Pathology, Beijing Institute of Radiation Medicine, Beijing 100850; ${ }^{3}$ Department of Stomatology, Chinese PLA General Hospital, Beijing 100853; ${ }^{4}$ Department of Oncology, State Key Discipline of Cell Biology, Xijing Hospital,

The Fourth Military Medical University, Xi'an 710032; ${ }^{5}$ State Key Laboratory of Proteomics, Beijing Proteome Research Center, Beijing Institute of Radiation Medicine, Beijing 100850, P.R. China

Received December 2, 2013; Accepted March 7, 2014

DOI: $10.3892 / \mathrm{ijmm} .2014 .1707$

\begin{abstract}
Forkhead box M1 (FoxM1) transcription factor is related to the pathogenesis of various malignancies and recent evidence indicates that FoxM1 promotes epithelialmesenchymal transition (EMT) in breast cancer. Metformin can inhibit the progression of cancer. However, whether FoxM1 plays a role in EMT in prostate cancer ( $\mathrm{PCa}$ ) and whether metformin can suppress EMT through FoxM1 in PCa remain unresolved issues. In this study, we investigated the expression levels of the FoxM1 protein in $62 \mathrm{PCa}$ and 39 benign prostate hyperplasia (BPH) samples and found that the expression levels of FoxM1 were higher in the PCa tissues (66.1\%) compared with the BPH tissues $(28.2 \%)(\mathrm{p}<0.05)$. We observed that FoxM1 was expressed in the PCa cell lines and that metformin suppressed cell proliferation and the expression of FoxM1. We induced EMT in the PCa cells by the addition of transforming growth factor (TGF)- $\beta 1$ and verified the process by examining EMT-related gene (E-cadherin, vimentin and Slug) expression. In addition, the knockdown of FoxM1 by shRNA in the PCa cells reversed EMT and markedly reduced cell migration. These results indicate that metformin suppresses EMT by inhibiting FoxM1. We demonstrate that the suppression of FoxM1 may be
\end{abstract}

Correspondence to: Professor Jie Tang, Department of Ultrasound, Chinese PLA General Hospital, 28 Fuxing Road, Beijing 100853, P.R. China

E-mail: txiner@vip.sina.com

Professor Lingqiang Zhang, State Key Laboratory of Proteomics, Beijing Proteomics Research Center, Beijing Institute of Radiation Medicine, 27 Taiping Road, Beijing 100850, P.R. China

E-mail: zhanglq@nic.bmi.ac.cn

*Contributed equally

Key words: forkhead box M1, prostate cancer, epithelial-mesenchymal transition, metformin, migration an effective therapeutic strategy for $\mathrm{PCa}$ and provide further evidence of the anticancer effects of metformin.

\section{Introduction}

Prostate cancer (PCa) is one of the most frequently diagnosed tumor types and its incidence increases with age in males worldwide. According to the latest evidence, among American males, the incidence rate of three types of cancer is considered to reach up to almost half that of all newly diagnosed cancers. These three types of cancer are prostate, lung and bronchus and colorectal cancer. The number of patients who suffer from PCa is 238,590 , which accounts for $28 \%$ of incident cases in males (1). As one of the critical mechanisms of tumor metastasis, epithelial-mesenchymal transition (EMT) is becoming a focus of research. EMT is characterized by the loss of epithelial markers and is accompanied by the increased expression of mesenchymal genes. The most important role of EMT in cancer cells is increased cell motility. It has been previously reported that in clinical prostate tumor specimens, EMT is not only a morphological change, but also a behavioral change (2).

Forkhead Box M1 (FoxM1) is a member of the large family of forkhead box (Fox) transcription factors which share a conserved winged helix DNA binding domain (3). A great deal of evidence has confirmed that FoxM1 is upregulated in various human malignancies, including breast cancer, lung cancer, ovarian carcinoma, hepatocellular carcinoma (HCC), pancreatic cancer, stomach cancer, non-Hodgkin's lymphoma, melanoma and colorectal cancer (4-12). In addition, according to recent evidence, the overexpression of FoxM1 correlates with poor prognosis in breast cancer, HCC and lung cancer (13-15). FoxM1 plays crucial roles in cell proliferation, cell cycle regulation, angiogenesis, invasion and metastasis (3,6,16-21). Recently, two studies indicated that FoxM1 is associated with EMT in lung fibrosis and breast cancer $(22,23)$. However, the differential expression of FoxM1 in benign and malignant prostate tissues and the role of FoxM1 in EMT in PCa cells have not yet been elucidated. 
Metformin has been widely used for the treatment of type 2 diabetic patients from the 1970's in Europe and from 1995 in the United States. In recent years, clinical and epidemiological evidence indicates that metformin is a novel anticancer agent (24). The potential mechanisms of action of metformin in cancer include the regulation of the cell cycle through the activation of AMP-activated protein kinase (AMPK), as well as its effects on tyrosine kinases and insulin (25). However, whether metformin can suppress EMT by regulating FoxM1 remains an unresolved issue.

In this study, we investigated the expression of FoxM1 in benign and malignant prostate tissues, as well as its correlation with clinicopathological characteristics. We further demonstrate that metformin inhibits EMT in PCa by downregulating FoxM1.

\section{Materials and methods}

Human tissue specimens and immunohistochemical analysis. The expression of FoxM1 was analyzed in a total of 101 human $\mathrm{PCa}$ and benign prostate hyperplasia (BPH) specimens (62 PCa and $39 \mathrm{BPH}$ ) from patients who were scheduled for prostate biopsy at the General Hospital of The Chinese People's Liberation Army (PLA; Beijing, China) between January 2009 and December 2013. The use of the tissue specimens was approved by the Ethics Committee of the Chinese PLA General Hospital. A histopathological analysis of the specimens was performed by pathologists at the Department of Pathology, General Hospital of The Chinese PLA. The results from immunohistochemistry were analyzed by two independent pathologists blinded to the clinical parameters. The scores of the staining results were based on the following criteria as described in a previous study (26): i) percentage of positive tumor cells in the tumor tissue: 0 (0\%), 1 (1-10\%), 2 (11-50\%), $3(51-70 \%)$ and 4 (71-100\%); ii) staining intensity: 0 (none), 1 (weak), 2 (moderate) and 3 (strong). The staining index was calculated as follows: staining intensity score $\mathrm{x}$ proportion of positive tumor cells. A final score of $\geq 5$ was considered as a high expression.

Cell culture. The human LNCaP PCa cell line was purchased from the American Type Culture Collection (ATCC; Rockville, MD, USA). The human DU145 PCa cell line was a gift from Professor J.G. Zhou from the Beijing Institute of Biotechnology (Beijing, China). The human PCa cell lines, PC3 and PC3M, were provided by Dr Wang Yu from the Department of Stomatology (Chinese PLA General Hospital). The DU145 and LNCaP cells were maintained in RPMI-1640 medium (Gibco, Grand Island, NY, USA) supplemented with 10\% FBS (HyClone Laboratories, Inc., Logan,UT, USA); the PC3 and PC3M cells were cultured in DMEM medium (Gibco) supplemented with $10 \%$ FBS. All the cells were maintained in a humidified atmosphere of $95 \%$ air plus $5 \% \mathrm{CO}_{2}$ at $37^{\circ} \mathrm{C}$.

Transforming growth factor (TGF)- $\beta$-induced EMT. For the induction of EMT, the cells were plated on the previous day. Following starvation (in serum-free medium) overnight, $10 \mathrm{ng} / \mathrm{ml}$ TGF- $\beta 1$ (R\&D Systems, Inc., Minneapolis, MN, USA) were added; the cells were then examined at various time points following treatment.
Table I. Primers for quantitative PCR.

\begin{tabular}{|c|c|}
\hline Gene & Primers \\
\hline E-cadherin & $\begin{array}{ll}\mathrm{F} & \text { 5'-CGGGAATGCAGTTGAGGATC-3' } \\
\mathrm{R} & \text { 5'-AGGATGGTGTAAGCGATGGC-3' }\end{array}$ \\
\hline Vimentin & $\begin{array}{ll}\text { F } & \text { 5'-GAGAACTTTGCCGTTGAAGC-3' } \\
\text { R } & \text { 5'-GCTTCCTGTAGGTGGCAATC-3' }\end{array}$ \\
\hline Snail1 & $\begin{array}{ll}\text { F } & \text { 5'-TCGGAAGCCTAACTACAGCGA-3' } \\
\text { R } & \text { 5'-AGATGAGCATTGGCAGCGAG-3' }\end{array}$ \\
\hline Snail2 (Slug) & $\begin{array}{ll}\text { F 5'- GATGCCGCGCTCCTTCCTGG-3' } \\
\text { R 5'-GGGGGACTCACTCGCCCCAA-3' }\end{array}$ \\
\hline Zeb1 & $\begin{array}{l}\text { F 5'- GATGATGAATGCGAGTCAGATGC-3' } \\
\text { R 5'- ACAGCAGTGTCTTGTTGTTGT-3' }\end{array}$ \\
\hline Zeb2 & $\begin{array}{ll}\text { F } & \text { 5'- CAAGAGGCGCAAACAAGCC-3' } \\
\text { R } & \text { 5'- GGTTGGCAATACCGTCATCC-3' }\end{array}$ \\
\hline FoxM1 & $\begin{array}{ll}\text { F } & \text { 5'- TTGGACCAGGTGTTTAAGCAGCAG-3' } \\
\text { R } & \text { 5'- GAGGAGTCTGCTGGGAACGGGAG-3' }\end{array}$ \\
\hline GAPDH & $\begin{array}{ll}\text { F } & \text { 5'-GTCATCCATGA-CAACTTTGG-3' } \\
\text { R } & \text { 5'-GAGCTTGACAAAGTGGTCGT-3' }\end{array}$ \\
\hline
\end{tabular}

FoxM1, forkhead box M1; Zeb, zinc finger E-box binding homeobox; F, forward; R, reverse.

Drug treatments and shRNA plasmid transfection. The cells were treated with metformin (Sigma-Aldrich, St. Louis, MO, USA). The cells were transfected with a FoxM1 shRNA plasmid (provided by Dr Wang Yu) (15) using Lipofectamine ${ }^{\mathrm{TM}} 2000$ (Invitrogen Life Technologies, Carlsbad, CA, USA).

RNA isolation, reverse-transcription and quantitative PCR. The DU145 cells were treated with the plasmid or metformin, followed by exposure to TGF- $\beta 1$. Subsequently, total RNA was prepared using TRIzol reagent (Invitrogen). Quantitative PCR was carried out as previously described (27). The primers were used for quantitative PCR are presented in Table I. They were synthesized by Sangon Biotech (Shanghai, China).

MTS assay. The cells were seeded at 1,000-5,000 cells per well (triplicates) in 96-well plates. After $24 \mathrm{~h}$, the cells were treated with metformin. A total of $20 \mu \mathrm{l}$ of MTS solution (Promega Corp., Madison, WI, USA) was added to each well, and the cells were incubated at $37^{\circ} \mathrm{C}$ with $5 \% \mathrm{CO}_{2}$ for $4 \mathrm{~h}$. Cell viability was detected by scanning with a microplate reader (Bio-Rad, Hercules, CA, USA) at $490 \mathrm{~nm}$.

Wound-healing assay. The cells were plated in 12-well culture plates in complete culture medium and grown to confluence. A wound was created by scraping with a sterilized $10 \mu \mathrm{l}$ pipette tip in the middle of the cell monolayer. The cells were then cultured with fresh complete culture medium containing $10 \mathrm{ng} / \mathrm{ml}$ TGF- $\beta 1$ with or without metformin treatment for $24 \mathrm{~h}$. Subsequently, the ability of the cells to migrate into the cleared section was observed and photographed using a microscope (Nikon Eclipse TS100; Nikon, Tokyo, Japan). 
Transwell assay. The migration ability of the cells was detected using 24-well plates with 8- $\mu \mathrm{m}$ pore size inserts (Corning Life Sciences, Oneonta, NY, USA). Following starvation overnight, $1 \times 10^{5}$ cells were added to the upper well in $200 \mathrm{ml}$ RPMI-1640 medium without FBS and allowed to migrate to the bottom compartment containing RPMI-1640 medium with 10\% FBS for $24 \mathrm{~h}$, followed by wiping off the non-migrated cells with a cotton swab. For the quantification of migration, Transwell filters were fixed in methanol for $10 \mathrm{~min}$ and stained with $0.1 \%$ crystal violet in $20 \%(\mathrm{v} / \mathrm{v})$ methanol for $20 \mathrm{~min}$ and mounted on a glass slide. The evaluation of the completed transmigration was performed under a microscope (x200 magnification). An equal volume of $10 \%$ acetic acid was then added to each well to completely dissolve the stained crystal violet. The OD value was detected by scanning with a microplate reader (Bio-Rad) at $570 \mathrm{~nm}$ to quantify the percentage of migrated cells. The experiments were performed in triplicate wells. Data are presented as the means $\pm \mathrm{SD}$ from three independent experiments.

Western blot analysis and reagents. Protein samples were size fractionated by SDS-PAGE and transferred onto PVDF membranes (Millipore UK Ltd., Consett, UK). The blots were blocked for $1 \mathrm{~h}$ in 5\% milk/0.1\% Tween-20 in TBS (TBS-T) and then incubated with primary antibodies at $4^{\circ} \mathrm{C}$ overnight. Western bolt analysis was conducted using anti-E-cadherin, anti-vimentin (Cell Signaling Technology, Inc., Danvers, MA, USA), anti-FoxM1, anti-Slug (Abcam, Cambridge, UK) and anti-p-AMPK $\alpha$ antibodies (Santa Cruz Biotechnology, Inc., Santa Cruz, CA, USA).

Statistical analysis. The correlations between the expression levels of FoxM1 and clinical parameters were evaluated by the $\chi^{2}$-test. Statistical analyses were performed using ANOVA or the Student's t-test. All analyses were performed using SPSS 12.0 software for Windows. P-values $<0.05$ were considered to indicate statistically significant differences.

\section{Results}

FoxM1 protein is upregulated in PCa tissues. To investigate the role of FoxM1 in the progression of $\mathrm{PCa}, 39 \mathrm{BPH}$ specimens and $62 \mathrm{PCa}$ specimens were collected in this study (Table II). The mean age of the patients with BPH was $61.4 \pm 13.4$ years. All BPH specimens showed histologically epithelial and stromal cell hyperplasia. There were also three cases accompanied with prostatic intraepithelial neoplasia (PIN) of grade I and four cases with PIN of grade II-III. Among the 62 patients suffering from $\mathrm{PCa}$, the mean age was $68.6 \pm 9.7$ years. The numbers of cases with Gleason scores of $<7$ and $\geq 7$ were 16 and 46, respectively. By immunohistochemical analysis, the expression levels of FoxM1 were mainly observed in the cytoplasm and the nucleus of the cells (Fig. 1A). The differential expression of FoxM1 was observed between the BPH and PCa tissues (Fig. 1B). The intensity scores of FoxM1 expression in the $\mathrm{PCa}$ tissues were markedly higher than those in the BPH tissues. In the three PIN cases of grade I and the four PIN cases of grade II-III, the number of cases with high levels of FoxM1 expression was one and three, respectively. Moreover, there was a positive correlation between the expression levels of FoxM1 and the Gleason score. The
Table II. Correlation between the protein expression of FoxM1 and clinicopathological parameters of the patients with prostate cancer.

\begin{tabular}{lcccc}
\hline & & \multicolumn{2}{c}{ FoxM1 expression } & \\
\cline { 3 - 4 } Characteristic & No. & Low & High & P-value \\
\hline BPH & 39 & $28(71.8 \%)$ & $11(28.2 \%)$ & $0.0005^{\mathrm{a}}$ \\
Age & & & & 0.768 \\
$\quad<60$ & 12 & 9 & 3 & \\
$\geq 60$ & 27 & 19 & 8 & \\
PIN & & & & \\
Grade I & 3 & 2 & 1 & \\
Grade II-III & 4 & 1 & 3 & \\
PCa & 62 & $21(33.9 \%)$ & $41(66.1 \%)$ & \\
Age & & & & 0.694 \\
$<60$ & 13 & 5 & 8 & \\
$\geq 60$ & 49 & 16 & 33 & \\
Gleason scores & & & & \\
$<7$ & 16 & 10 & 6 & \\
$\geq 7$ & 46 & 11 & 35 &
\end{tabular}

FoxM1, Forkhead box M1; BPH, benign prostate hyperplasia; PIN, prostatic

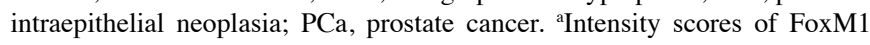
expression in $\mathrm{BPH}$ compared with those in $\mathrm{PCa}$ tissues.

results indicated that FoxM1 expression levels were higher in the tissues with a Gleason score $\geq 7$ compared with those with a Gleason score $<7$. These results indicate that FoxM1 plays a crucial role in the progression of PCa (Fig. 1C).

Elevated endogenous expression of FoxM1 protein in $\mathrm{PCa}$ cell lines. To explore the endogenous expression of FoxM1 in PCa cells, the expression of FoxM1 in four human PCa cell lines was examined by quantitative RT-PCR and western blot analysis. The PCa cell lines (LNCaP, DU-145, PC-3 and PC-3M) showed a positive expression of FoxM1 by western blot analysis (Fig. 2A). The mRNA levels of FoxM1 were detected in the aforementioned cell lines by quantitative PCR (Fig. 2B). Furthermore, a FoxM1 plasmid was transfected into the LNCaP PCa cells and a FoxM1 shRNA plasmid was transfected into the DU-145 PCa cells (Fig. 2C). The results revealed good transfection efficiencies.

Metformin suppresses the proliferation of PCa cells and downregulates the protein expression of FoxM1. To determine whether metformin inhibits the proliferation of PCa cells and in particular, the expression of FoxM1 in PCa cells, the growth curves and expression of FoxM1 were observed in the PCa cells treated with metformin. In the DU-145 and LNCaP cells, cell viability was markedly inhibited by metformin (Fig. 3A). The expression levels of FoxM1 protein in the DU-145 cells markedly decreased when the cells were treated with metformin in a time- and dose-dependent manner (Fig. 3B). Consistent with previous findings (28), metformin as an AMPK activator, decreased the expression of FoxM1. Thus, these data 
A

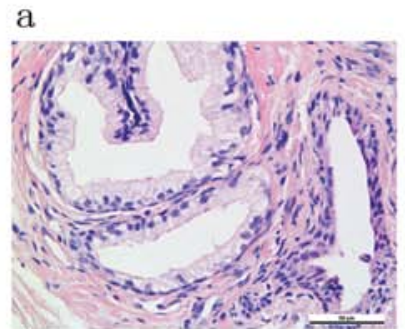

C

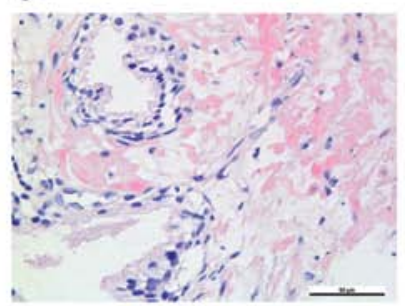

B

a

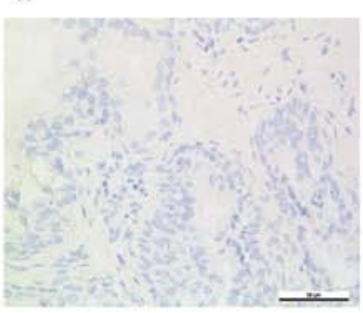

b

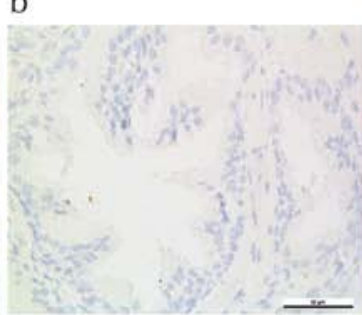

e

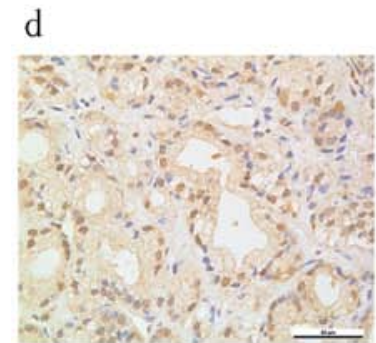

$f$

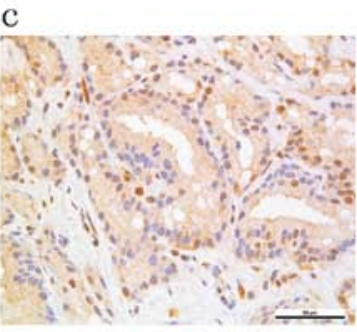

$\mathrm{f}$

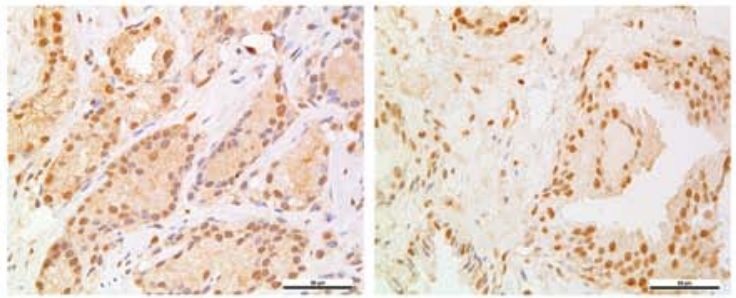

C

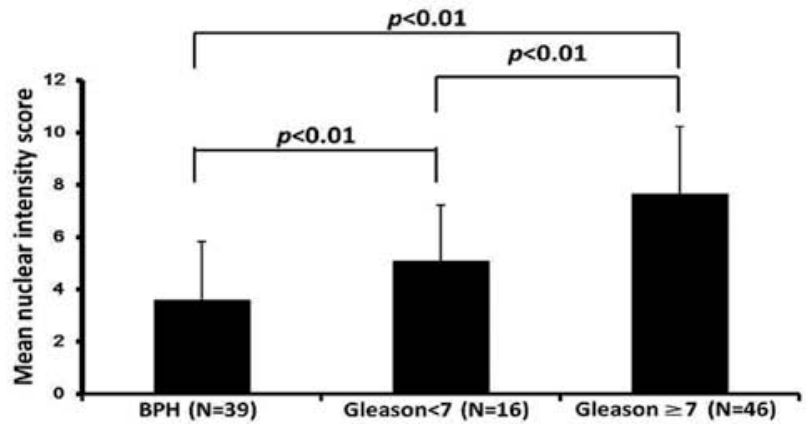

Figure 1. Differential expression of forkhead box M1 (FoxM1) in benign and malignant prostate tissues. (A) Representative images of hematoxylin and eosin staining and immunohistochemical staining of FoxM1 protein in (a and b) benign prostate hyperplasia (BPH) and (c and d) prostate cancer (PCa) tissues. (B) Representation of FoxM1 protein expression in prostate tissues. Different staining intensities of FoxM1 protein from (a-f) are (a and b) negative, (c and d) weak, (e) moderate and (f) strong. (C) Differential FoxM1 expression between BPH and PCa tissues. FoxM1 expression levels are high in PCa tissues compared with those in BPH tissues ( $<<0.01$ ). Between the groups with a Gleason score $<7$ and $\geq 7$, the FoxM1 expression levels differ ( $<<0.01$ ). The results indicated that FoxM1 expression is associated with the progression of PCa.

demonstrate that metformin suppresses the proliferation and downregulates the expression of FoxM1 in PCa cells.

Establishment of in vitro model of EMT using PCa cell lines. In order to examine the association between FoxM1 and
EMT in PCa cells, the establishment of an in vitro model of EMT is essential. TGF- $\beta 1(10 \mathrm{ng} / \mathrm{ml})$ stimulation was used to induce EMT in the DU-145 cells in our study. Following treatment with TGF- $\beta 1$ for $24 \mathrm{~h}$, the cell morphology was slightly altered (Fig. 4A). The epithelial cancer cells were 
A

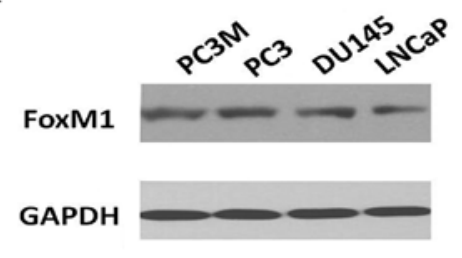

$\mathrm{C}$

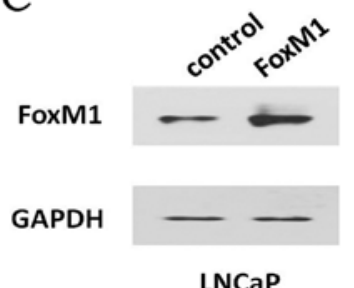

B
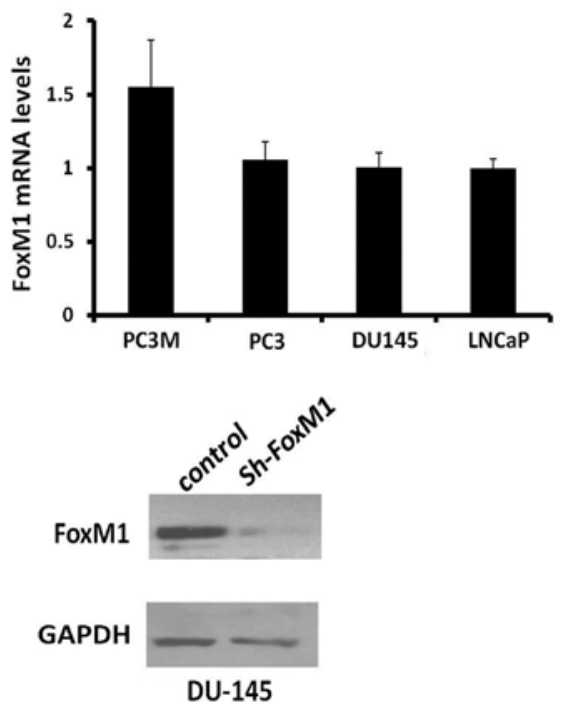

Figure 2. Expression of forkhead box M1 (FoxM1) protein in prostate cancer (PCa) cell lines. (A) FoxM1 protein is expressed in PCa cell lines (PC-3, PC-3M DU-145 and LNCaP). (B) FoxM1 mRNA levels in PCa cell lines. The expression is slightly higher in the PC-3M cells,although there is no significant difference among the four cell lines. (C) Transfection with plasmid of FoxM1 in LNCaP cells and FoxM1 shRNA in DU-145 cells.

A

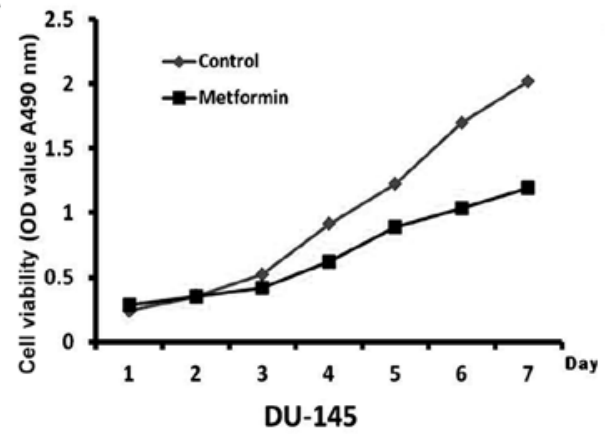

B

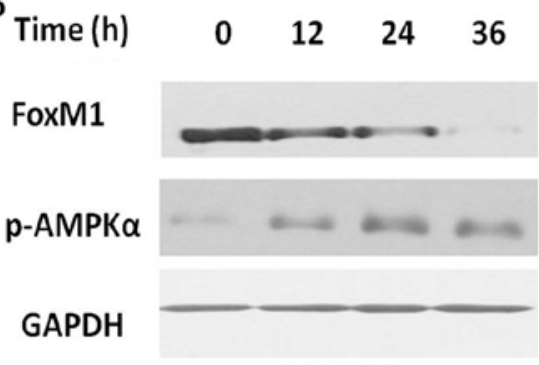

DU-145
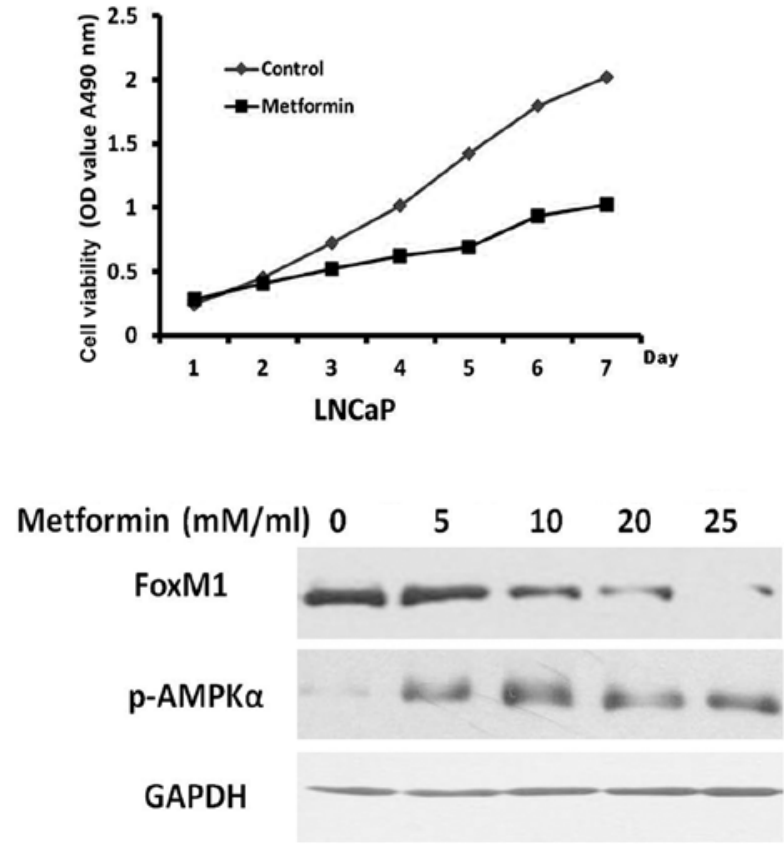

DU-145

Figure 3. Metformin suppresses cell proliferation and the expression of forkhead box M1 (FoxM1) in prostate cancer (PCa). (A) The growth curves are lower in the metformin $(20 \mathrm{mM})$-treated groups compared with the control groups. The cell proliferation of DU-145 and LNCaP cells was reduced in a time-dependent manner. (B) Following treatment with metformin $(20 \mathrm{mM})$ for different periods of time, the expression levels of FoxM1 decreased accompanied by the increased p-AMPK $\alpha$ levels. The effects of metformin on FoxM1 also occurred in a dose-dependent manner.

partly transformed into mesenchymal-like cells. The epithelial marker, E-cadherin, and the mesenchymal markers, vimentin and Snail2 (Slug), were also examined by western blot analysis (Fig. 4B). The protein expression of E-cadherin was downregulated, whereas the expression of vimentin and Slug was upregulated. The results indicated that the model of EMT with the DU-145 cells was established. The mRNA levels of EMT-related genes were also detected (Fig. 4C); the mRNA level of E-cadherin was decreased, while the mRNA levels of vimentin and Slug were increased.

Metformin prevents TGF- $\beta 1$-induced EMT through FoxM1. In order to examine whether FoxM1 is required for the migration of PCa cells, FoxM1 shRNA was transfected into the DU-145 cells. After 24 h, TGF- $\beta 1$ was added to the cells. The protein levels of E-cadherin, vimentin and Slug were examined by 
A

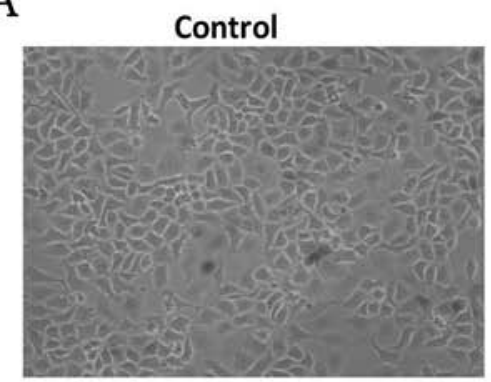

DU-145
TGF- $\beta 10 \mathrm{ng} / \mathrm{ml} 24 \mathrm{~h}$

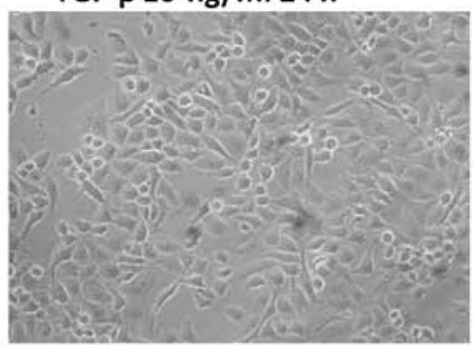

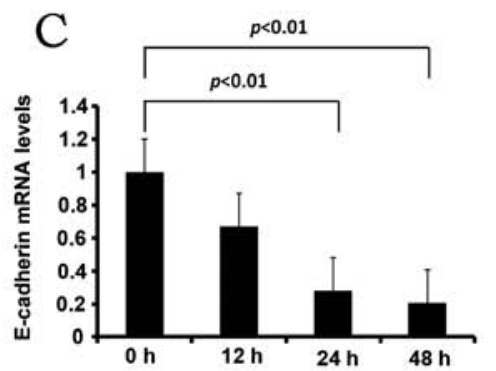

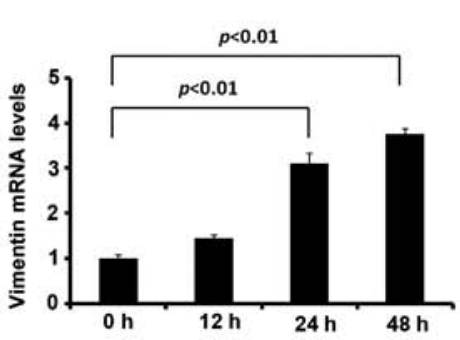

$\mathrm{B}$
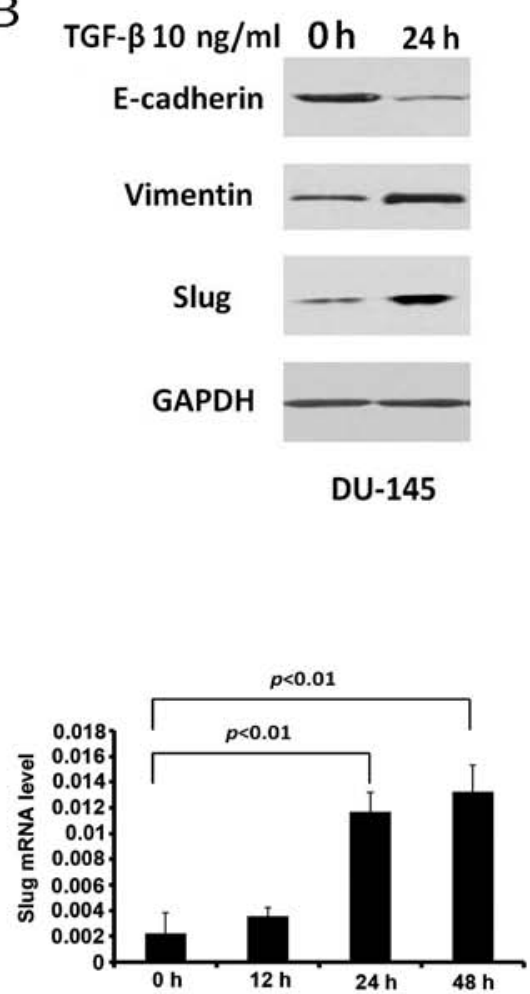

Figure 4. TGF- $\beta 1$ induces epithelial-mesenchymal transition (EMT) in prostate cancer (PCa) cell lines in vitro. (A) The morphology of the epithelial DU-145 cells was altered. The cells transformed into mesenchymal-like cells following treatment with TGF- $\beta 1$ (10 ng/ml) $24 \mathrm{~h}$. (B) Western blot analysis revealed that the protein levels of EMT-related molecular were changed following treatment with TGF- $\beta 1$. The expression level of E-cadherin protein decreased and that of vimentin and Slug increased. This suggested that EMT occurred in the PCa cell lines. (C) The same results were also verified by the mRNA levels. The results of quantitative PCR indicated that following induction with TGF- $\beta 1(10 \mathrm{ng} / \mathrm{ml})$, the mRNA levels of E-cadherin, vimentin and Slug were altered in a time-dependent manner.

western blot analysis. The protein level of E-cadherin was increased partly, whereas the expression levels of vimentin and Slug in the cells transfected with FoxM1 shRNA were decreased (Fig. 5A). The mRNA levels of FoxM1, E-cadherin, vimentin, Snail1, Slug, zinc finger E-box binding homeobox (Zeb)1 and Zeb2 in the FoxM1-knockdown cells were also examined by quantitative PCR. The loss of FoxM1 was associated with the increased E-cadherin mRNA and the decreased vimentin, Slug and Zeb2 mRNA expression (Fig. 5B).

To confirm that FoxM1 regulates PCa cell migration, the migration ability of the DU-145 cells was detected following transfection with FoxM1 shRNA. The wound disappeared after $24 \mathrm{~h}$ in the control cells, while the self-healing ability of the FoxM1 shRNA-transfected cells was poor (Fig. 5C). After plating into the insert of the Transwell chamber ( $24 \mathrm{~h}$ later), the cell migration ability was measured. The knockdown of FoxM1 markedly decreased the migration ability of the DU145 cells (Fig. 5D). These data confirm that FoxM1 is required for the migration of $\mathrm{PCa}$ cells and that metformin regulates the EMT process in PCa cells through FoxM1.

\section{Discussion}

PCa is one of the human malignant cancers of which the incidence and mortality rate are very high. In particular, in patients with advanced stages of PCa, metastasis will aggravate the condition and shorten the life span of the patients. In recent years, the process of EMT in cancer has increasingly become a research hotspot. As previously demonstrated, in pathological specimens, the altered expression of various cell lineage markers supports the hypothesis that the EMT process promotes the progression of PCa (29).

FoxM1 belongs to the forkhead superfamily of transcription factors which share an evolutionary conserved 'winged helix' DNA-binding domain. It regulates the expression of downstream target genes by the consensus binding sequence, TAAACA $(30,31)$. FoxM1 is expressed in proliferating cells, but its expression is lost in cells which are in the stationary phase and are terminally differentiated. It plays important roles in cell proliferation, cell cycle, cell differentiation, angiogenesis and metastasis. Previous studies have demonstrated that FoxM1 is upregulated in diverse human malignancies and its high expression indicates poor prognosis $(4,8,11,14,15)$. Although the expression of FoxM1 in PCa has been previously reported (7), our study compares the expression of FoxM1 in $\mathrm{BPH}$ with that in PCa tissues obtained from the Chinese population. The results revealed that the expression level of FoxM1 protein was highly elevated in the PCa tissues. Our data also demonstrated that FoxM1 was expressed in the BPH tissues. However, the intensity scores of FoxM1 expression were markedly higher in the PCa tissues and were associated with the Gleason scores. According to these results, the high protein expression of FoxM1 may be a potential marker indicating the progression of PCa. 


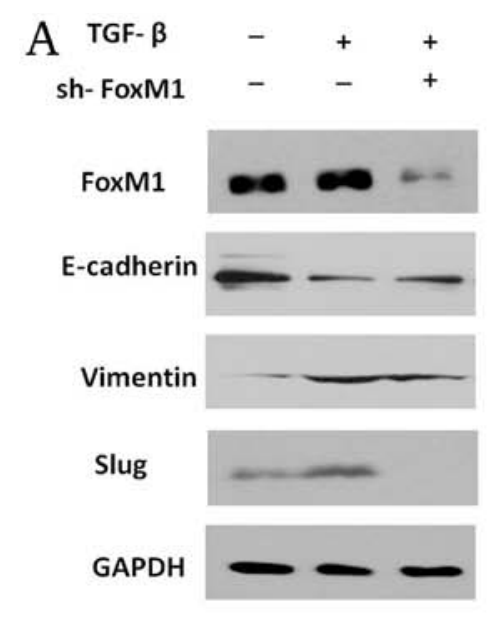

B

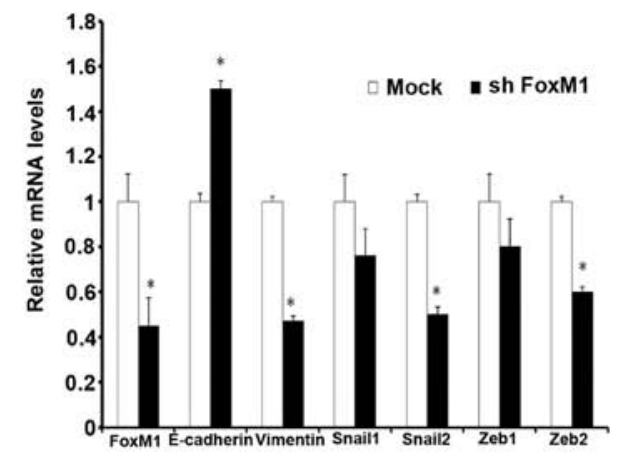

DU-145

C

Control

sh-FoxM1
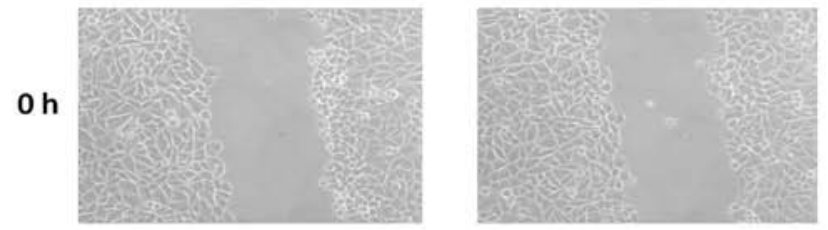

$24 \mathrm{~h}$
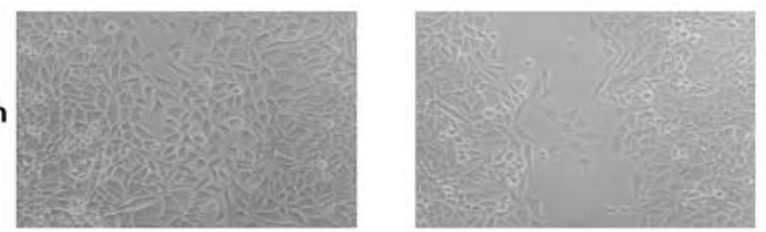

DU-145

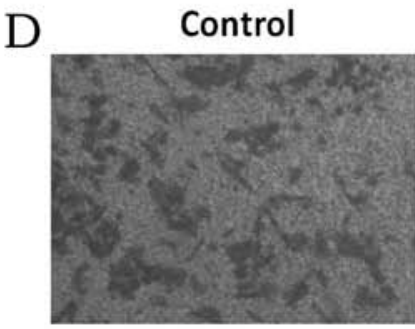

sh-FoxM1
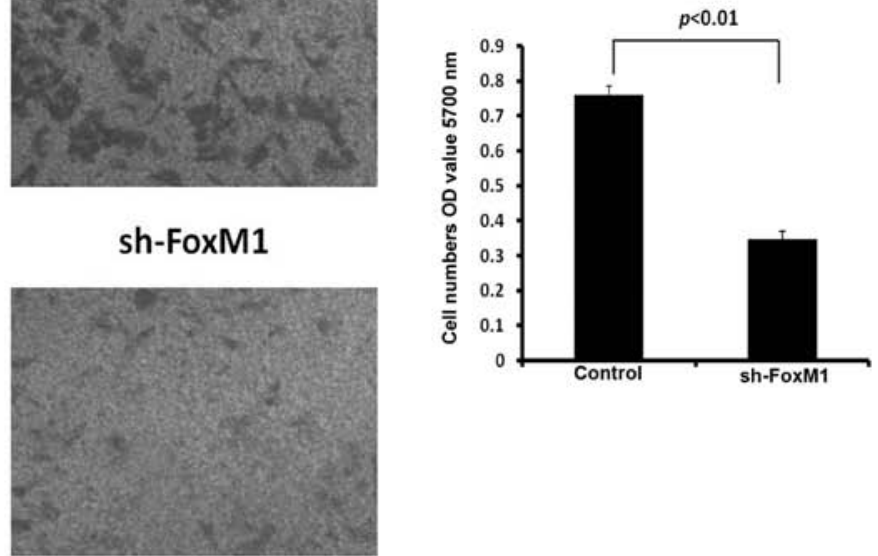

Figure 5. Metformin inhibits epithelial-mesenchymal transition (EMT) through forkhead box M1 (FoxM1) in prostate cancer (PCa) cell lines. (A) The knockdown of FoxM1 by shRNA reversed EMT in PCa cells. The decreased E-cadherin protein increased following transfection with FoxM1 shRNA. The elevated expression of vimentin and Slug protein was reduced. (B) Quantitative PCR revealed that the mRNA levels of EMT-related genes in the DU-145 cells transfected with FoxM1 shRNA were altered. The mRNA levels of E-cadherin increased, while those of vimentin, Slug and Zeb2 were significantly decreased $(\mathrm{p}<0.01)$. (C and D) Downregulation of FoxM1 significantly reduced DU-145 cell migration. After $24 \mathrm{~h}$ of TGF- $\beta 1$ (10 ng/ml) induction, the wounds were not healed in the FoxM1 shRNA group. The number of cells that migrated through the membranes was fewer than that of the control group ( $<<0.01)$.

EMT is detected initially in embryonic development and wound healing in physiological processes. Subsequently,
EMT is known to be associated with fibrotic diseases and cancer (32). EMT is classified into three subtypes according 
to the biological features (33). EMT during implantation, embryogenesis, and organ development is defined as type 1. Type 2 refers to EMT related to tissue regeneration and organ fibrosis. A previous study reported that EMT found in prostate hyperplasia cells should belong to this type (34). Type 3 , which we payed close attention to in our study, occurs in the process of cancer progression and metastasis. The changes that occur in biomarkers in EMT can be summed up in two parts: loss of epithelial cell markers, such as E-cadherin and cytokeratins, and the upregulation of mesenchymal cell markers, including vimentin and $\mathrm{N}$-cadherin (29). Several transcription factors have been found to involved in the process of EMT, such as the Snail family of zinc-finger transcription factors, Snail and Slug; the two-handed zinc-finger factors of $\delta E F 1$ family proteins, Zeb1 and Zeb2 (also known as Smad-interacting protein), and the basic helix-loop-helix factor, Twist $(33,35,36)$. Further studies have shown that the Snail transcription factor inhibits E-cadherin expression by binding several E-boxes located in the promoter region (37). In this study, our data indicated that FoxM1 was involved in the process of EMT induced by TGF- $\beta$ in PCa cells. Our results are in accordance with those of a previous study, in which Slug was regulated by FoxM1 (23). These results strengthen the concept that the Snail family plays an important role in EMT in cancer. FoxM1 is a crucial transcription factor in EMT by regulating the Snail family.

Evidence from epidemiological, histopathological, molecular pathological and clinical studies indicates that there is a correlation between metabolic syndrome (MetS) and the the development of BPH and PCa $(38,39)$. As the most common therapy to lower blood glucose concentration in patients with type 2 diabetes and MetS, metformin notably blocks hepatic glucose production, reduces insulin resistance and lowers insulin levels. Several studies have shown that it inhibits human cancer cell proliferation and tumor growth by decreasing cyclin D1 expression (40). In addition to this, metformin has been proven to impede the TGF- $\beta$-induced loss of the epithelial marker, E-cadherin, in human breast cancer cells (41). In our study, we found that metformin inhibited the expression of FoxM1; thus, it participates in the regulation of EMT in PCa cells. Our results are in accordance with those presented in the study by Yung et al (28); thus, the suppression of FoxM1 by metformin is dependent on the AKT/FOXO3a/FoxM1 signaling cascade in PCa cells. When FoxM1 was knocked down, the expression of the gene, Slug, which is crucial for EMT, was markedly decreased. Thus, these results indicate that metformin inhibits EMT through the downregulation of FoxM1 expression in PCa. However, there were some limitations in our study. Firstly, the number of specimens was limited and a larger sample size is required to further confirm our results. Secondly, due to the limited research time, the dynamic tracing data was not sufficient. We aim to provide further information and discuss the correlation between the expression of FoxM1 and the outcome of PCa patients in future studies.

In conclusion, the data in the current study suggest that the expression levels of FoxM1 are associated with the progression of PCa. FoxM1 plays an important role in EMT in PCa and the suppressive effects of metformin on EMT may partly involve the downregulation of FoxM1. FoxM1 plays a crucial role in the progression of PCa and the inhibition of FoxM1 may prove to be an effective therapeutic strategy.

\section{Acknowledgements}

We thank Professor J.G. Zhou for providing the DU-145 cell line. This study was supported in part by a grant from the National Natural Science Foundation of China (no. 81171357).

\section{References}

1. Siegel R, Naishadham D and Jemal A: Cancer statistics, 2013. CA Cancer J Clin 63: 11-30, 2013.

2. Christiansen JJ: Reassessing epithelial to mesenchymal transition as a prerequisite for carcinoma invasion and metastasis. Cancer Res 66: 8319-8326, 2006.

3. Laoukili J, Kooistra MRH, Brás A, et al: FoxM1 is required for execution of the mitotic programme and chromosome stability. Nat Cell Biol 7: 126-136, 2005.

4. Yang DK, Son CH, Lee SK, Choi PJ, Lee KE and Roh MS: Forkhead box M1 expression in pulmonary squamous cell carcinoma: correlation with clinicopathologic features and its prognostic significance. Hum Pathol 40: 464-470, 2009.

5. Kretschmer C, Sterner-Kock A, Siedentopf F, Schoenegg W, Schlag PM and Kemmner W: Identification of early molecular markers for breast cancer. Mol Cancer 10: 15, 2011.

6. Wang Z, Banerjee S, Kong D, Li Y and Sarkar FH: Downregulation of Forkhead Box M1 transcription factor leads to the inhibition of invasion and angiogenesis of pancreatic cancer cells. Cancer Res 67: 8293-8300, 2007.

7. Kalin TV, Wang IC, Ackerson TJ, Major ML, Detrisac CJ, Kalinichenko VV, Lyubimov A and Costa RH: Increased levels of the FoxM1 transcription factor accelerate development and progression of prostate carcinomas in both TRAMP and LADY transgenic mice. Cancer Res 66: 1712-1720, 2006.

8. Sun HC, Li M, Lu JL, et al: Overexpression of Forkhead box M1 protein associates with aggressive tumor features and poor prognosis of hepatocellular carcinoma. Oncol Rep 25: 1533-1539, 2011.

9. Cancer Genome Atlas Research Network: Integrated genomic analyses of ovarian carcinoma. Nature 474: 609-615, 2011.

10. Green MR, Aya-Bonilla C, Gandhi MK, et al: Integrative genomic profiling reveals conserved genetic mechanisms for tumorigenesis in common entities of non-Hodgkin's lymphoma. Genes Chromosomes Cancer 50: 313-326, 2011.

11. Huynh KM, Soh JW, Dash R, Sarkar D, Fisher PB and Kang D: FOXM1 expression mediates growth suppression during terminal differentiation of HO-1 human metastatic melanoma cells. J Cell Physiol 226: 194-204, 2011.

12. Uddin S, Ahmed M, Hussain A, Abubaker J, Al-Sanea N, AbdulJabbar A, Ashari LH, Alhomoud S, Al-Dayel F, Jehan Z, Bavi P, Siraj AK and Al-Kuraya KS: Genome-wide expression analysis of Middle Eastern colorectal cancer reveals FOXM1 as a novel target for cancer therapy. Am J Pathol 178: 537-547, 2011.

13. Bektas N1, Haaf At, Veeck J, et al: Tight correlation between expression of the Forkhead transcription factor FOXMl and HER2 in human breast cancer. BMC Cancer 8: 42, 2008.

14. Xia L1, Mo P, Huang W, Zhang L, Wang Y, Zhu H, Tian D, Liu J, Chen Z, Zhang Y, Chen Z, Hu H, Fan D, Nie Y and Wu K: The TNF- $\alpha /$ ROS/HIF-1-induced upregulation of FoxMI expression promotes HCC proliferation and resistance to apoptosis. Carcinogenesis 33: 2250-2259, 2012.

15. Wang Y1, Wen L, Zhao SH, Ai ZH, Guo JZ and Liu WC: FoxM1 expression is significantly associated with cisplatin-based chemotherapy resistance and poor prognosis in advanced non-small cell lung cancer patients. Lung Cancer 79: 173-179, 2013.

16. Wang IC, Chen YJ, Hughes D, et al: Forkhead box M1 regulates the transcriptional network of genes essential for mitotic progression and genes encoding the SCF (Skp2-Cks1) ubiquitin ligase. Mol Cell Biol 25: 10875-10894, 2005.

17. Ahmad A, Wang Z, Kong D, et al: FoxM1 down-regulation leads to inhibition of proliferation, migration and invasion of breast cancer cells through the modulation of extra-cellular matrix degrading factors. Breast Cancer Res Treat 122: 337-346, 2010.

18. Priller M, Pöschl J, Abrão L, et al: Expression of FoxM1 is required for the proliferation of medulloblastoma cells and indicates worse survival of patients. Clin Cancer Res 17: 6791-6801, 2011.

19. Li Q, Zhang N, Jia Z, et al: Critical role and regulation of transcription factor FoxM1 in human gastric cancer angiogenesis and progression. Cancer Res 69: 3501-3509, 2009. 
20. Karadedou CT, Gomes AR, Chen J, et al: FOXO3a represses VEGF expression through FOXM1-dependent and -independent mechanisms in breast cancer. Oncogene 31: 1845-1858, 2012

21. Dai B, Kang SH, Gong W, et al: Aberrant FoxM1B expression increases matrix metalloproteinase-2 transcription and enhances the invasion of glioma cells. Oncogene 26: 6212-6219, 2007.

22. Balli D, Ustiyan V, Zhang Y, et al: Foxml transcription factor is required for lung fibrosis and epithelial-to-mesenchymal transition. EMBO J 32: 231-244, 2013.

23. Yang $\mathrm{C}$, Chen $\mathrm{H}$, Tan $\mathrm{G}$, et al: FOXM1 promotes the epithelial to mesenchymal transition by stimulating the transcription of Slug in human breast cancer. Cancer Lett 340: 104-112, 2013.

24. Del Barco S, Vazquez-Martin A, Cufi S, et al: Metformin: multifaceted protection against cancer. Oncotarget 2: 896-917, 2011.

25. Hadad SM, Hardie DG, Appleyard V and Thompson AM Effects of metformin on breast cancer cell proliferation, the AMPK pathway and the cell cycle. Clin Transl Oncol: Dec 12, 2013 (Epub ahead of print).

26. He SY, Shen HW, Xu L, et al: FOXM1 promotes tumor cell invasion and correlates with poor prognosis in early-stage cervical cancer. Gynecol Oncol 127: 601-610, 2012.

27. Lu K, Yin X, Weng T, et al: Targeting WW domains linker of HECT-type ubiquitin ligase Smurf1 for activation by CKIP-1 Nat Cell Biol 10: 994-1002, 2008.

28. Yung MM CD, Liu VW, Yao KM and Ngan HY: Activation of AMPK inhibits cervical cancer cell growth through AKT FOXO3a FOXM1 signaling cascade. BMC Cancer 13: 3272013.

29. Hugo H, Ackland ML, Blick T, et al: Epithelial-mesenchymal and mesenchymal-epithelial transitions in carcinoma progression. J Cell Physiol 213: 374-383, 2007.

30. Korver W, Roose J, Heinen K, et al: The human TRIDENT/ HFH-11/FKHL16 gene: structure, localization, and promoter characterization. Genomics 46: 435-442, 1997.

31. Laoukili J, Stahl M and Medema RH: FoxM1: at the crossroads of ageing and cancer. Biochim Biophys Acta 1775: 92-102, 2007.
32. Nieto MA: Epithelial-mesenchymal transitions in development and disease: old views and new perspectives. Int J Dev Biol 53 1541-1547, 2009

33. Kalluri R and Weinberg RA: The basics of epithelial-mesenchymal transition. J Clin Invest 119: 1420-1428, 2009.

34. Slabáková E, Pernicová Z, Slavíčková E, Staršíchová A, Kozubík A and Souček K: TGF- $\beta 1$-induced EMT of non-transformed prostate hyperplasia cells is characterized by early induction of SNAI2/Slug. Prostate 71: 1332-1343, 2011.

35. Katsuno Y, Lamouille S and Derynck R: TGF- $\beta$ signaling and epithelial-mesenchymal transition in cancer progression. Curr Opin Oncol 25: 76-84, 2013.

36. Saitoh M and Miyazawa K: Transcriptional and post-transcriptional regulation in TGF- $\beta$-mediated epithelial-mesenchymal transition. J Biochem 151: 563-571, 2012.

37. Lin T, Ponn A, Hu X, Law BK and Lu J: Requirement of the histone demethylase LSD1 in Snail-mediated transcriptional repression during epithelial-mesenchymal transition. Oncogene 29: 4896-4904, 2010.

38. Gorbachinsky I, Akpinar H and Assimos DG: Metabolic syndrome and urologic diseases. Rev Urol 12: e157-e180, 2010.

39. Parsons JK, Carter HB, Partin AW, et al: Metabolic factors associated with benign prostatic hyperplasia. J Clin Endocrinol Metab 91: 2562-2568, 2006

40. Jalving M, Gietema JA, Lefrandt JD, et al: Metformin: taking away the candy for cancer? Eur J Cancer 46: 2369-2380, 2010.

41. Cufi S, Vazquez-Martin A, Oliveras-Ferraros C, MartinCastillo B, Joven J and Menendez JA: Metformin against TGF $\beta$-induced epithelial-to-mesenchymal transition (EMT): from cancer stem cells to aging-associated fibrosis. Cell Cycle 9: 4461-4468, 2010. 